\title{
On local spectral properties of operator matrices
}

\author{
II Ju An ${ }^{1}$, Eungil Ko ${ }^{2}$ and Ji Eun Lee ${ }^{3^{*}}$
}

\author{
"Correspondence: \\ jieunlee7@sejong.ac.kr; \\ jieun7@ewhain.net \\ ${ }^{3}$ Department of Mathematics and \\ Statistics, Sejong University, Seoul, \\ 143-747, Korea \\ Full list of author information is \\ available at the end of the article
}

\begin{abstract}
In this paper, we focus on a $2 \times 2$ operator matrix $T_{\epsilon_{k}}$ as follows:$$
T_{\epsilon_{k}}=\left(\begin{array}{cc}
A & C \\
\epsilon_{k} D & B
\end{array}\right)
$$

where $\epsilon_{k}$ is a positive sequence such that $\lim _{k \rightarrow \infty} \epsilon_{k}=0$. We first explore how $T_{\epsilon_{k}}$ has several local spectral properties such as the single-valued extension property, the property $(\beta)$, and decomposable. We next study the relationship between some spectra of $T_{\epsilon_{k}}$ and spectra of its diagonal entries, and find some hypotheses by which $T_{\epsilon_{k}}$ satisfies Weyl's theorem and $a$-Weyl's theorem. Finally, we give some conditions that such an operator matrix $T_{\epsilon_{k}}$ has a nontrivial hyperinvariant subspace.
\end{abstract}

MSC: 47A10;47A11;47A15; 47A53

Keywords: $2 \times 2$ operator matrices; Hyperinvariant subspace; The single-valued extension property; The property $(\beta)$; Decomposable; Weyl's theorem

\section{Springer}

\section{Introduction}

Let $\mathcal{L}(\mathcal{H})$ denote the algebra of bounded linear operators on a separable Hilbert space $\mathcal{H}$. Let $\{T\}^{\prime}$, the commutant of $T$, be the collection of all bounded linear operators such that commute with $T$. A subspace $\mathcal{G} \subset \mathcal{H}$ is invariant for $T \in \mathcal{L}(\mathcal{H})$ if an inclusion $T \mathcal{G} \subset$ $\mathcal{G}$ holds, and is hyperinvariant for $T$ if the inclusion $S \mathcal{G} \subset \mathcal{G}$ holds for all $S \in\{T\}^{\prime}$. The hyperinvariant subspace problem is asking whether every operator on a separable complex Hilbert space has a nontrivial hyperinvariant subspace. It has been known that this is one of unresolved problems in operator theory and it has attracted a lot of interest by many authors.

For the study of this problem, in 2011, H. J. Kim [8] proved that, if $T=\left(\begin{array}{cc}T_{1} & T_{2} \\ 0 & T_{3}\end{array}\right) \in$ $\mathcal{L}(\mathcal{H} \oplus \mathcal{H})$ where $T_{1}, T_{2}$, and $T_{3}$ are arbitrary operators in $\mathcal{L}(\mathcal{H})$ such that $T_{1}$ is either a compact operator with $T_{1} \neq 0$ or a normal operator with $T_{1} \neq \lambda I$, then at least one of $T$ and $\widehat{T}$, has a nontrivial hyperinvariant subspace where $\widehat{T}=\left(\begin{array}{cc}T_{3} & T_{4} \\ 0 & T_{1}\end{array}\right)$ for an arbitrary operator $T_{4} \in \mathcal{L}(\mathcal{H})$. In 2018, I. B. Jung, E. Ko, and C. Pearcy [7] showed if $T_{1}$ and $T_{3}$ are operators in $\mathcal{L}(\mathcal{H})$ such that either $T_{1}$ or $T_{3}$ has a nontrivial hyperinvariant subspace, then $T$ and $\widehat{T}$ have a nontrivial hyperinvariant subspace where $T_{2}$ and $T_{4}$ are any operators in

(c) The Author(s) 2021. This article is licensed under a Creative Commons Attribution 4.0 International License, which permits use, sharing, adaptation, distribution and reproduction in any medium or format, as long as you give appropriate credit to the original author(s) and the source, provide a link to the Creative Commons licence, and indicate if changes were made. The images or other third party material in this article are included in the article's Creative Commons licence, unless indicated otherwise in a credit line to the material. If material is not included in the article's Creative Commons licence and your intended use is not permitted by statutory regulation or exceeds the permitted use, you will need to obtain permission directly from the copyright holder. To view a copy of this licence, visit http://creativecommons.org/licenses/by/4.0/. 
$\mathcal{L}(\mathcal{H})$. As mentioned in the above results, in the case of a $2 \times 2$ upper triangular operator matrix, there are some known results, but in the case of a full $2 \times 2$ operator matrix, it is very difficult to solve the invariant subspace problem. So, we focus on the matrix $T_{\epsilon_{k}}$ as a variation of the $2 \times 2$ upper triangular operator matrix and we study some conditions so that a $2 \times 2$ operator matrix $T_{\epsilon_{k}}$ has a nontrivial hyperinvariant subspace.

We now provide a simple outline of the paper. We first study the local spectral theory of operator matrices (cf. [3] and [9]). In particular, we consider the case when the $(2,1)$ entry of a $2 \times 2$ operator matrix approaches zero. In addition, we give the relationship between some spectra of $2 \times 2$ operator matrices and spectra of their diagonal entries, and find some hypotheses by which such operator matrices $T_{\epsilon_{k}}$ entail Weyl's theorem and $a$-Weyl's theorem.

\section{Preliminaries}

We briefly review some notions of local spectral properties, which are used in this paper. We refer to [10] for more detailed information.

The operator $T \in \mathcal{L}(\mathcal{H})$ has the single-valued extension property if $f(\lambda) \equiv 0$ is the unique solution to $(T-\lambda) f(\lambda) \equiv 0$ on $D$ for every open subset $D$ of $\mathbb{C}$ and any $\mathcal{H}$-valued analytic function $f$ on $D$. The local resolvent set $\rho_{T}(x)$ of $T \in \mathcal{L}(\mathcal{H})$ at $x \in \mathcal{H}$ is the union of all open subset $D$ of $\mathbb{C}$ such that there is an analytic function $f: D \rightarrow \mathcal{H}$ such that $(T-\lambda) f(\lambda) \equiv x$ on $D$. The set $\sigma_{T}(x)=\mathbb{C} \backslash \rho_{T}(x)$ is the local spectrum of $T$ at $x$. The local spectral subspace of an operator $T \in \mathcal{L}(\mathcal{H})$ is given by $H_{T}(F)=\left\{x \in \mathcal{H}: \sigma_{T}(x) \subset F\right\}$ for any $F \subset \mathbb{C}$. We say that $T \in \mathcal{L}(\mathcal{H})$ has Bishop's property $(\beta)$ if every sequence $\left\{f_{n}\right\}$ of $\mathcal{H}$-valued analytic functions on $D$ for every open subset $D$ of $\mathbb{C}$ such that $(T-\lambda) f_{n}(\lambda)$ converges uniformly to 0 in norm on compact subsets of $D$, it follows that $f_{n}(\lambda)$ converges uniformly to 0 in norm on compact subsets of $G$. Notice that, if $T$ has Bishop's property $(\beta)$, then it has the singlevalued extension property. The operator $T \in \mathcal{L}(\mathcal{H})$ is decomposable if for every open cover $\{U, V\}$ of $\mathbb{C}$ there are $T$-invariant subspaces $\mathcal{M}$ and $\mathcal{N}$ such that

$$
\mathcal{H}=\mathcal{M}+\mathcal{N}, \quad \sigma\left(\left.T\right|_{\mathcal{M}}\right) \subset \bar{U} \quad \text { and } \quad \sigma\left(\left.T\right|_{\mathcal{N}}\right) \subset \bar{V} .
$$

In general, it is known that $T$ is decomposable if and only if $T$ and its adjoint $T^{*}$ possess property $(\beta)[1,10]$.

Now, we introduce some Weyl type theorems related to definitions of various spectra (see [12] for more details). For these, we first take a look at some notions needed in this paper. If $T \in \mathcal{L}(\mathcal{H})$, we shall write $\operatorname{ker}(T)$ (or $N(T)$ ) and $\operatorname{ran}(T)$ (or $R(T)$ ) for the null space and the range of $T$, respectively. We know that the family $\left\{\operatorname{ker}\left(T^{k}\right)\right\}$ forms an ascending sequence of subspaces for $T \in \mathcal{L}(\mathcal{H})$ and $k \in \mathbb{N}$. So we call the ascent of $T$ for the smallest nonnegative integer $k$ for which $\operatorname{ker}\left(T^{k}\right)=\operatorname{ker}\left(T^{k+1}\right)$ holds. We also see that the family $\left\{\operatorname{ran}\left(T^{k}\right)\right\}$ forms a descending sequence for $k \in \mathbb{N}$, and then the smallest nonnegative integer $k$ for which $\operatorname{ran}\left(T^{k}\right)=\operatorname{ran}\left(T^{k+1}\right)$ is said the descent of $T$. An operator $T \in \mathcal{L}(\mathcal{H})$ is called upper semi-Fredholm (resp., lower semi-Fredholm) if it has both finite dimensional kernel and closed range (resp., it has both finite dimensional co-kernel and closed range). Either upper or lower semi-Fredholm operator $T \in \mathcal{L}(\mathcal{H})$ is called semi-Fredholm, and its index is given by $\operatorname{ind}(T):=\operatorname{dim} \operatorname{ker}(T)-\operatorname{dim} \operatorname{ker}\left(T^{*}\right)$. When both $\operatorname{dim} \operatorname{ker}(T)$ and $\operatorname{dim} \operatorname{ker}\left(T^{*}\right)$ are finite, then $T$ is called Fredholm. If $T \in \mathcal{L}(\mathcal{H})$ is a Fredholm operator satisfying $\operatorname{ind}(T)=0$, then it is called Weyl, and if $T$ is a Fredholm operator with finite descent and ascent, then it is called Browder. 
If $T \in \mathcal{L}(\mathcal{H})$, we shall write $\sigma_{p}(T), \sigma_{s}(T), \sigma_{a}(T), \sigma(T), \sigma_{e}(T), \sigma_{l e}(T)$, and $\sigma_{r e}(T)$ the point spectrum, the surjective spectrum, the approximate point spectrum, the spectrum, the essential spectrum, the left essential spectrum, and the right essential spectrum the left essential spectrum of $T$, respectively. The Weyl spectrum $\sigma_{w}(T):=\{\mu \in \mathbb{C}: T-\mu I$ is not Weyl $\}$ and the Browder spectrum $\sigma_{b}(T):=\{\mu \in \mathbb{C}: T-\mu I$ is not Browder $\}$, where $I$ is an identity operator on $\mathcal{H}$. We write $\mathcal{K}(\mathcal{H})$ for the set of all compact operators on $\mathcal{H}$ and review another spectra as follows: the Weyl essential approximate point spectrum $\sigma_{e a}(T):=$ $\{\mu \in \mathbb{C}: T+C-\mu I$ is not bounded below for all $C \in \mathcal{K}(\mathcal{H})\}$ and the Browder essential approximate point spectrum $\sigma_{a b}(T):=\{\mu \in \mathbb{C}: T+C-\mu I$ is not bounded below for all $C \in$ $\mathcal{K}(\mathcal{H})$ and $T C=C T\}$. Evidently, we get the inclusions

$$
\sigma_{e}(T) \subseteq \sigma_{w}(T) \subseteq \sigma_{b}(T) \text { and } \quad \sigma_{e a}(T) \subseteq \sigma_{a b}(T)
$$

Let iso $K$ be the collection of all isolated points of a complex subset $K$. We write $\pi_{00}(T):=$ $\{\lambda \in \operatorname{iso} \sigma(T): 0<\operatorname{dim} \operatorname{ker}(T-\lambda)<\infty\}$. And we denote $p_{00}(T):=\sigma(T) \backslash \sigma_{b}(T)$ which is the collection of Riesz points of T. We say that Weyl's theorem is obeyed for $T$ provided $\sigma(T) \backslash \sigma_{w}(T)=\pi_{00}(T)$, and that Browder's theorem is obeyed for $T$ provided $\sigma(T) \backslash \sigma_{w}(T)=$ $p_{00}(T)$, equivalently, if $\sigma_{w}(T)=\sigma_{b}(T)$. We say that $a$-Weyl's theorem is obeyed for $T$ provided $\sigma_{a}(T) \backslash \sigma_{e a}(T)=\pi_{00}^{a}(T)$ and that a-Browder's theorem is obeyed for $T$ provided $\sigma_{a}(T) \backslash \sigma_{e a}(T)=p_{00}^{a}(T)$, where $\pi_{00}^{a}(T):=\left\{\lambda \in\right.$ iso $\left.\sigma_{a}(T): 0<\operatorname{dim} \operatorname{ker}(T-\lambda)<\infty\right\}$ and $p_{00}^{a}(T):=\sigma_{a}(T) \backslash \sigma_{a b}(T)$. Then it is well known that

$$
a \text {-Weyl's theorem } \Longrightarrow a \text {-Browder's theorem }
$$

$$
\Downarrow
$$

Weyl's theorem $\Longrightarrow$ Browder's theorem

\section{Main results}

In this section, we study $2 \times 2$ operator matrices. In particular, we consider the case when their $(2,1)$-entry approaches zero. We begin our program with the following theorem.

Theorem 3.1 Let $T_{\epsilon_{k}}=\left(\begin{array}{cc}A & C \\ \epsilon_{k} D & B\end{array}\right)$ where $A, B, C, D \in \mathcal{L}(\mathcal{H})$ and $\left\{\epsilon_{k}\right\}$ is a positive sequence such that $\lim _{k \rightarrow \infty} \epsilon_{k}=0$. Then the following statements hold.

(i) If both $A$ and $B$ have the single-valued extension property, then $T_{\epsilon_{k}}$ has the single-valued extension property.

(ii) If $T_{\epsilon_{k}}$ has the single-valued extension property, $B C=C B$, and $C$ is nilpotent of order $m$, then $B$ has the single-valued extension property.

Proof (i) Suppose that $A$ and $B$ have the single-valued extension property. Let $G$ be an open set in $\mathbb{C}$ and let $f: G \rightarrow \mathcal{H} \oplus \mathcal{H}$ be an analytic function with $f=f_{1} \oplus f_{2}$ such that

$$
\left(T_{\epsilon_{k}}-\lambda\right)\left(\begin{array}{l}
f_{1}(\lambda) \\
f_{2}(\lambda)
\end{array}\right)=0 .
$$

Then

$$
\left(\begin{array}{cc}
A-\lambda & C \\
\epsilon_{k} D & B-\lambda
\end{array}\right)\left(\begin{array}{l}
f_{1}(\lambda) \\
f_{2}(\lambda)
\end{array}\right)=\left(\begin{array}{l}
0 \\
0
\end{array}\right) .
$$


Therefore, we get

$$
\left\{\begin{array}{l}
(A-\lambda) f_{1}(\lambda)+C f_{2}(\lambda)=0, \\
\epsilon_{k} D f_{1}(\lambda)+(B-\lambda) f_{2}(\lambda)=0 .
\end{array}\right.
$$

Since $\left\|(B-\lambda) f_{2}(\lambda)\right\| \leq\left\|\epsilon_{k} D f_{1}(\lambda)+(B-\lambda) f_{2}(\lambda)\right\|+\epsilon_{k}\left\|D f_{1}(\lambda)\right\|=\epsilon_{k}\left\|D f_{1}(\lambda)\right\|$ and $\lim _{k \rightarrow \infty} \epsilon_{k}=$ $0,(B-\lambda) f_{2}(\lambda)=0$. Moreover, since $B$ has the single-valued extension property, $f_{2}(\lambda)=0$. From (2), we have

$$
(A-\lambda) f_{1}(\lambda)=0
$$

Since $A$ has the single-valued extension property, $f_{1}(\lambda)=0$. Hence $T_{\epsilon_{k}}$ has the singlevalued extension property.

(ii) Let $T_{\epsilon_{k}}$ have the single-valued extension property and $(B-\lambda) f_{2}(\lambda)=0$ where $f_{2}$ is an analytic function. Then

$$
\left(T_{\epsilon_{k}}-\lambda\right)\left(\begin{array}{c}
0 \\
C^{m-1} f_{2}(\lambda)
\end{array}\right)=\left(\begin{array}{l}
0 \\
0
\end{array}\right) .
$$

Since $T_{\epsilon_{k}}$ has the single-valued extension property, it follows from (3) that $C^{m-1} f_{2}(\lambda)=0$. Thus

$$
\left(T_{\epsilon_{k}}-\lambda\right)\left(\begin{array}{c}
0 \\
C^{m-2} f_{2}(\lambda)
\end{array}\right)=\left(\begin{array}{l}
0 \\
0
\end{array}\right) .
$$

Since $T_{\epsilon_{k}}$ has the single-valued extension property, $C^{m-2} f_{2}(\lambda)=0$. By induction, we have $f_{2}(\lambda)=0$. Hence $B$ has the single-valued extension property.

Corollary 3.2 Let $T_{\epsilon_{k}}=\left(\begin{array}{cc}A & C \\ \epsilon_{k} D & B\end{array}\right)$ where $A, B, C, D \in \mathcal{L}(\mathcal{H})$ and $\left\{\epsilon_{k}\right\}$ is a positive sequence such that $\lim _{k \rightarrow \infty} \epsilon_{k}=0$. If $A$ and $B$ have the single-valued extension property, then the following inclusions hold.

(i) $\sigma_{B}\left(x_{2}\right) \subset \sigma_{T_{\epsilon_{k}}}\left(x_{1} \oplus x_{2}\right)$ for all $x_{1}, x_{2} \in \mathcal{H}$ and $H_{T_{\epsilon_{k}}}(F) \subset \mathcal{H} \oplus H_{B}(F)$ for any subset $F$ of $\mathbb{C}$.

(ii) $\sigma_{A}\left(x_{1}\right) \subset \sigma_{T_{\epsilon_{k}}}\left(x_{1} \oplus 0\right)$ for all $x_{1} \in \mathcal{H}$.

Proof (i) We know that $T_{\epsilon_{k}}$ has the single-valued extension property from Theorem 3.1. Let $\lambda_{0} \notin \sigma_{T_{\epsilon_{k}}}\left(x_{1} \oplus x_{2}\right)$ for all $x_{1}, x_{2} \in \mathcal{H}$. Then there exists a neighborhood $\mathcal{D}$ of $\lambda_{0}$ and an analytic function $f=f_{1} \oplus f_{2}: \mathcal{D} \rightarrow \mathcal{H} \oplus \mathcal{H}$ such that $\left(T_{\epsilon_{k}}-\lambda\right) f(\lambda)=x_{1} \oplus x_{2}$ for every $\lambda \in \mathcal{D}$. Then we have

$$
\left\{\begin{array}{l}
(A-\lambda) f_{1}(\lambda)+C f_{2}(\lambda)=x_{1}, \\
\epsilon_{k} D f_{1}(\lambda)+(B-\lambda) f_{2}(\lambda)=x_{2} .
\end{array}\right.
$$

Letting $\epsilon_{k} \rightarrow 0,(B-\lambda) f_{2}(\lambda)=x_{2}$. Hence $\lambda_{0} \notin \sigma_{B}\left(x_{2}\right)$ for all $x_{2} \in \mathcal{H}$.

On the other hand, if $x_{1} \oplus x_{2} \in H_{T_{\epsilon_{k}}}(F)$, then $\sigma_{T_{\epsilon_{k}}}\left(x_{1} \oplus x_{2}\right) \subset F$. Since

$$
\sigma_{B}\left(x_{2}\right) \subset \sigma_{T_{\epsilon_{k}}}\left(x_{1} \oplus x_{2}\right) \subset F,
$$


it follows from (i) that $x_{2} \in H_{B}(F)$. Thus $x_{1} \oplus x_{2} \in \mathcal{H} \oplus H_{B}(F)$. Hence

$$
H_{T_{\epsilon_{k}}}(F) \subset \mathcal{H} \oplus H_{B}(F)
$$

for any subset $F$ of $\mathbb{C}$.

(ii) Let $\lambda_{0} \in \rho_{T_{\epsilon_{k}}}\left(x_{1} \oplus 0\right)$ for all $x_{1} \in \mathcal{H}$. Then there exists a neighborhood $\mathcal{G}$ of $\lambda_{0}$ and an analytic function $f_{1} \oplus f_{2}: \mathcal{G} \rightarrow \mathcal{H} \oplus \mathcal{H}$ such that $\left(T_{\epsilon_{k}}-\lambda\right)\left(\begin{array}{c}f_{1}(\lambda) \\ f_{2}(\lambda)\end{array}\right)=\left(\begin{array}{c}x_{1} \\ 0\end{array}\right)$ for every $\lambda \in \mathcal{G}$. Thus this implies that

$$
\left\{\begin{array}{l}
(A-\lambda) f_{1}(\lambda)+C f_{2}(\lambda)=x_{1} \\
\epsilon_{k} D f_{1}(\lambda)+(B-\lambda) f_{2}(\lambda)=0
\end{array}\right.
$$

Letting $\epsilon_{k} \rightarrow 0$, we get $(B-\lambda) f_{2}(\lambda)=0$. Since $B$ has the single-valued extension property, $f_{2}(\lambda)=0$ for every $\lambda \in \mathcal{G}$. Thus $(A-\lambda) f_{1}(\lambda)=x_{1}$, and hence $\lambda_{0} \in \rho_{A}\left(x_{1}\right)$. Therefore $\sigma_{A}\left(x_{1}\right) \subset$ $\sigma_{T_{\epsilon_{k}}}\left(x_{1} \oplus 0\right)$ for all $x_{1} \in \mathcal{H}$.

Example 3.3 In Corollary 3.2, if both $A$ and $B$ are substituted with the unilateral shift $U$ on $\ell^{2}(\mathbb{N})$, then $T_{\epsilon_{k}}$ has the single-valued extension property on $\ell^{2}(\mathbb{N}) \oplus \ell^{2}(\mathbb{N})$. Furthermore, we get the following inclusions:

$$
\sigma_{U}\left(x_{1}\right) \subset \sigma_{T_{\epsilon_{k}}}\left(x_{1} \oplus 0\right) \quad \text { and } \quad \sigma_{U}\left(x_{2}\right) \subset \sigma_{T_{\epsilon_{k}}}\left(x_{1} \oplus x_{2}\right)
$$

for all $x_{1}, x_{2} \in \ell^{2}(\mathbb{N})$.

We next investigate some relations among the spectra, the point spectra and the approximate point spectra of $A, B$ and $T_{\epsilon_{k}}$, respectively.

Theorem 3.4 Let $T_{\epsilon_{k}}=\left(\begin{array}{cc}A & C \\ \epsilon_{k} D & B\end{array}\right)$ where $A, B, C, D \in \mathcal{L}(\mathcal{H})$ and $\left\{\epsilon_{k}\right\}$ is a positive sequence such that $\lim _{k \rightarrow \infty} \epsilon_{k}=0$.

(i) If both $A$ and $B$ have the single-valued extension property, then

$$
\sigma(A) \cup \sigma(B)=\sigma\left(T_{\epsilon_{k}}\right) \quad \text { and } \quad \sigma_{p}(A) \cup \sigma_{p}(B)=\sigma_{p}\left(T_{\epsilon_{k}}\right)
$$

(ii) If both $A^{*}$ and $B^{*}$ have the single-valued extension property, then

$$
\sigma_{a}(A) \cup \sigma_{a}(B)=\sigma_{a}\left(T_{\epsilon_{k}}\right)
$$

Proof (i) From Theorem 3.1, we know that $T_{\epsilon_{k}}$ has the single-valued extension property. Since $\sigma_{B}\left(x_{2}\right) \subset \sigma_{T_{\epsilon_{k}}}\left(x_{1} \oplus x_{2}\right)$ from Corollary 3.2, we have

$$
\sigma(B)=\bigcup_{x_{2} \in \mathcal{H}} \sigma_{B}\left(x_{2}\right) \subset \bigcup_{x_{1} \oplus x_{2} \in \mathcal{H} \oplus \mathcal{H}} \sigma_{T_{\epsilon_{k}}}\left(x_{1} \oplus x_{2}\right)=\sigma\left(T_{\epsilon_{k}}\right) .
$$

Since $\sigma_{A}\left(x_{1}\right) \subset \sigma_{T_{\epsilon_{k}}}\left(x_{1} \oplus 0\right)$ for all $x_{1} \in \mathcal{H}$, we get

$$
\sigma(A)=\bigcup_{x_{1} \in \mathcal{H}} \sigma_{A}\left(x_{1}\right) \subset \bigcup_{x_{1} \oplus x_{2} \in \mathcal{H} \oplus \mathcal{H}} \sigma_{T_{\epsilon_{k}}}\left(x_{1} \oplus x_{2}\right)=\sigma\left(T_{\epsilon_{k}}\right) .
$$


For the converse, we suppose that $\gamma \notin \sigma(A) \cup \sigma(B)$. If $\lim _{n \rightarrow \infty}\left\|\left(T_{\epsilon_{k}}-\gamma\right)\left(\begin{array}{l}x_{n} \\ y_{n}\end{array}\right)\right\|=0$, then

$$
\left\{\begin{array}{l}
\lim _{n \rightarrow \infty}\left\|(A-\gamma) x_{n}+C y_{n}\right\|=0, \\
\lim _{n \rightarrow \infty}\left\|\epsilon_{k} D x_{n}+(B-\gamma) y_{n}\right\|=0 .
\end{array}\right.
$$

Since $B-\gamma$ is invertible,

$$
\begin{aligned}
\left\|y_{n}\right\| & \leq\left\|(B-\gamma)^{-1}\right\|\left\|(B-\gamma) y_{n}\right\| \\
& \leq\left\|(B-\gamma)^{-1}\right\|\left\|(B-\gamma) y_{n}+\epsilon_{k} D x_{n}\right\|+\epsilon_{k}\|D\|\left\|(B-\gamma)^{-1}\right\|\left\|x_{n}\right\| .
\end{aligned}
$$

Then $\limsup _{n \rightarrow \infty}\left\|y_{n}\right\| \leq \epsilon_{k}\|D\|\left\|(B-\gamma)^{-1}\right\|\left(\lim \sup _{n \rightarrow \infty}\left\|x_{n}\right\|\right)$. Taking $\lim _{k \rightarrow \infty} \epsilon_{k}=0$, we have $\lim \sup _{n \rightarrow \infty}\left\|y_{n}\right\|=0$ and so

$$
\lim _{n \rightarrow \infty}\left\|y_{n}\right\|=0
$$

From (5), $\lim _{n \rightarrow \infty}\left\|(A-\gamma) x_{n}\right\|=0$. Since $A-\gamma$ is invertible, $\lim _{n \rightarrow \infty}\left\|x_{n}\right\|=0$. Therefore, $T_{\epsilon_{k}}-\gamma$ is bounded below. If $\left(T_{\epsilon_{k}}-\gamma\right)^{*}\left(\begin{array}{l}x \\ y\end{array}\right)=\left(\begin{array}{l}0 \\ 0\end{array}\right)$, then

$$
\left\{\begin{array}{l}
\left(A^{*}-\bar{\gamma}\right) x+\epsilon_{k} D^{*} y=0 \\
C^{*} x+\left(B^{*}-\bar{\gamma}\right) y=0
\end{array}\right.
$$

Since $\lim _{k \rightarrow \infty} \epsilon_{k}=0$, we have $\left(A^{*}-\bar{\gamma}\right) x=0$. Since $A^{*}-\bar{\gamma}$ is invertible, $x=0$ and so ( $B^{*}-$ $\bar{\gamma}) y=0$. Since $B^{*}-\bar{\gamma}$ is invertible, $y=0$. Thus $\operatorname{ker}\left(T_{\epsilon_{k}}-\gamma\right)^{*}=\{0\}$ and $\operatorname{so} \operatorname{ran}\left(T_{\epsilon_{k}}-\gamma\right)$ is dense in $\mathcal{H} \oplus \mathcal{H}$. Hence $T_{\epsilon_{k}}-\gamma$ is invertible. So $\gamma \notin \sigma\left(T_{\epsilon_{k}}\right)$. Consequently, the first equation is established. Moreover, if $A$ has the single-valued extension property, then it is well known that the surjective spectrum $\sigma_{s}(A)$ of $A$ identifies with the spectrum of $A$ (see [10]), so that $\sigma_{p}(A)=\sigma(A) \backslash \sigma_{s}(A)=\emptyset$. Similarly, $\sigma_{p}(B)=\emptyset$. Hence $\sigma_{p}(A) \cup \sigma_{p}(B)=$ $\emptyset \subset \sigma_{p}\left(T_{\epsilon_{k}}\right)$. Since $T_{\epsilon_{k}}$ has the single-valued extension property by Theorem 3.1, we have $\sigma_{p}\left(T_{\epsilon_{k}}\right)=\sigma\left(T_{\epsilon_{k}}\right) \backslash \sigma_{s}\left(T_{\epsilon_{k}}\right)=\emptyset$. From these arguments, the second equality trivially holds.

(ii) Suppose that both $A^{*}$ and $B^{*}$ have the single-valued extension property. Then we can prove that $T_{\epsilon_{k}}^{*}$ also has the single-valued extension property using a similar method from the proof of Theorem 3.1. It is known that $\sigma_{a}(T)=\sigma(T)$ provided $T^{*}$ has the single-valued extension property for every $T \in \mathcal{L}(\mathcal{H})$. This means that the equality $\sigma_{a}(A) \cup \sigma_{a}(B)=$ $\sigma_{a}\left(T_{\epsilon_{k}}\right)$ holds by (i).

It is well known that, if $A$ and $B$ have the property $(\beta)$, then $\left(\begin{array}{ll}A & C \\ 0 & B\end{array}\right)$ has the property $(\beta)$ without any conditions. However, $2 \times 2$ operator matrices which their all entries are nonzero, in addition, their $(2,1)$-entries are either $\mu I$ for some nonzero constant $\mu$, or $\epsilon_{k} I$ for a positive sequence $\left\{\epsilon_{k}\right\}$ with $\lim _{k \rightarrow \infty} \epsilon_{k}=0$ may not have the property $(\beta)$ even though their diagonal entries have the property $(\beta)$ (see (8)). We now study the property $(\beta)$ and decomposability of such a $2 \times 2$ operator matrix $T_{\epsilon_{k}}$.

Theorem 3.5 Let $T_{\epsilon_{k}}=\left(\begin{array}{cc}A & C \\ \epsilon_{k} D & B\end{array}\right)$ where $A, B, C, D \in \mathcal{L}(\mathcal{H})$ and $\left\{\epsilon_{k}\right\}$ is a positive sequence such that $\lim _{k \rightarrow \infty} \epsilon_{k}=0$. If $\sup _{n}\left\|f_{n, 1}\right\|_{K}<\infty$ whenever

$$
\left\|\left(T_{\epsilon_{k}}-\lambda\right)\left(\begin{array}{l}
f_{n, 1}(\lambda) \\
f_{n, 2}(\lambda)
\end{array}\right)\right\|_{K} \rightarrow 0 \quad \text { as } n \rightarrow \infty
$$


then the following statements hold.

(i) If $A$ and $B$ have the property $(\beta)$, then $T_{\epsilon_{k}}$ has the property $(\beta)$.

(ii) If $A$ and $B$ are decomposable, then $T_{\epsilon_{k}}$ is decomposable.

Proof (i) Suppose that $A$ and $B$ have the property $(\beta)$. Let $G$ be an open set in $\mathbb{C}$ and let $f_{n}: G \rightarrow \mathcal{H} \oplus \mathcal{H}$ be a sequence of analytic functions with $f_{n}=f_{n, 1} \oplus f_{n, 2}$ such that

$$
\lim _{n \rightarrow \infty}\left\|\left(T_{\epsilon_{k}}-\lambda\right)\left(\begin{array}{l}
f_{n, 1}(\lambda) \\
f_{n, 2}(\lambda)
\end{array}\right)\right\|_{K}=0
$$

for every compact set $K$ in $G$, where $\|f\|_{K}=\sup _{\lambda \in K}\|f(\lambda)\|$ for an $\mathcal{H} \oplus \mathcal{H}$-valued function $f(\lambda)$. Then

$$
\lim _{n \rightarrow \infty}\left\|\left(\begin{array}{cc}
A-\lambda & C \\
\epsilon_{k} D & B-\lambda
\end{array}\right)\left(\begin{array}{l}
f_{n, 1}(\lambda) \\
f_{n, 2}(\lambda)
\end{array}\right)\right\|_{K}=\left(\begin{array}{l}
0 \\
0
\end{array}\right) .
$$

Therefore, we get

$$
\left\{\begin{array}{l}
\lim _{n \rightarrow \infty}\left\|(A-\lambda) f_{n, 1}(\lambda)+C f_{n, 2}(\lambda)\right\|_{K}=0, \\
\lim _{n \rightarrow \infty}\left\|\epsilon_{k} D f_{n, 1}(\lambda)+(B-\lambda) f_{n, 2}(\lambda)\right\|_{K}=0 .
\end{array}\right.
$$

We observe that

$$
\begin{aligned}
\left\|(B-\lambda) f_{n, 2}(\lambda)\right\|_{K} & \leq\left\|(B-\lambda) f_{n, 2}(\lambda)+\epsilon_{k} D f_{n, 1}(\lambda)-\epsilon_{k} D f_{n, 1}(\lambda)\right\|_{K} \\
& \leq\left\|(B-\lambda) f_{n, 2}(\lambda)+\epsilon_{k} D f_{n, 1}(\lambda)\right\|_{K}+\left\|\epsilon_{k} D f_{n, 1}(\lambda)\right\|_{K} .
\end{aligned}
$$

From (8), we get

$$
\limsup _{n \rightarrow \infty}\left\|(B-\lambda) f_{n, 2}(\lambda)\right\|_{K} \leq \epsilon_{k}\|D\| \limsup _{n \rightarrow \infty}\left\|f_{n, 1}(\lambda)\right\|_{K} .
$$

Since $\sup _{n}\left\|f_{n, 1}\right\|_{K}<\infty$, letting $\epsilon_{k} \rightarrow 0$,

$$
\limsup _{n \rightarrow \infty}\left\|(B-\lambda) f_{n, 2}(\lambda)\right\|_{K}=0
$$

and so $\lim _{n \rightarrow \infty}\left\|(B-\lambda) f_{n, 2}(\lambda)\right\|_{K}=0$. Moreover, since $B$ has the property $(\beta)$, $\lim _{n \rightarrow \infty}\left\|f_{n, 2}(\lambda)\right\|_{K}=0$. From (8), we have

$$
\lim _{n \rightarrow \infty}\left\|(A-\lambda) f_{n, 1}(\lambda)\right\|_{K}=0 .
$$

Since $A$ has the property $(\beta), \lim _{n \rightarrow \infty}\left\|f_{n, 1}(\lambda)\right\|_{K}=0$. Thus

$$
\lim _{n \rightarrow \infty}\left\|f_{n}(\lambda)\right\|_{K}=\lim _{n \rightarrow \infty}\left\|f_{n, 1}(\lambda) \oplus f_{n, 2}(\lambda)\right\|_{K}=0 .
$$

Hence $T_{\epsilon_{k}}$ has the property $(\beta)$.

(ii) If $A$ and $B$ are decomposable, then $A, A^{*}, B$, and $B^{*}$ have the property $(\beta)$. Since $A$ and $B$ have the property $(\beta)$, it follows from (1) that $T_{\epsilon_{k}}$ has the property $(\beta)$. Note that 
$T_{\epsilon_{k}}^{*}=\left(\begin{array}{cc}A^{*} & \epsilon_{k} D^{*} \\ C^{*} & B^{*}\end{array}\right)$ and $\left(\begin{array}{cc}0 & I \\ I & 0\end{array}\right)\left(\begin{array}{cc}B^{*} & C^{*} \\ \epsilon_{k} D^{*} & A^{*}\end{array}\right)\left(\begin{array}{ll}0 & I \\ I & 0\end{array}\right)=T_{\epsilon_{k}}^{*}$. Since $A^{*}$ and $B^{*}$ also have property $(\beta)$, it follows from (i) that $\left(\begin{array}{cc}B^{*} & C^{*} \\ \epsilon_{k} D^{*} & A^{*}\end{array}\right)$ has the property $(\beta)$. Since $\left(\begin{array}{cc}B^{*} & C^{*} \\ \epsilon_{k} D^{*} & A^{*}\end{array}\right)$ is unitarily equivalent to $T_{\epsilon_{k}}^{*}$, we see that $T_{\epsilon_{k}}^{*}$ has the property $(\beta)$. Therefore $T_{\epsilon_{k}}$ is decomposable.

From these arguments for some local spectral properties of the operator matrices $T_{\epsilon_{k}}$, we get more corollaries.

Corollary 3.6 Let $T_{\epsilon_{k}}=\left(\begin{array}{cc}A & C \\ \epsilon_{k} D & B\end{array}\right)$ where $A, B, C, D \in \mathcal{L}(\mathcal{H})$ and $\left\{\epsilon_{k}\right\}$ is a positive sequence such that $\lim _{k \rightarrow \infty} \epsilon_{k}=0$. If $\sup _{n}\left\|f_{n, 1}\right\|_{K}<\infty$ whenever (6), then the following statements are satisfied.

(i) If $A$ and $B$ have the property $(\beta)$ and $\sigma\left(T_{\epsilon_{k}}\right)$ has nonempty interior, then $T_{\epsilon_{k}}$ has a nontrivial invariant subspace.

(ii) If $A$ and $B$ are hyponormal, then $T_{\epsilon_{k}}$ has the property $(\beta)$.

(iii) If $A$ and $B$ are compact or normal, then $T_{\epsilon_{k}}$ is decomposable.

Proof (i) If $A$ and $B$ have the property $(\beta)$, then it follows from Theorem 3.5 that $T_{\epsilon_{k}}$ has the property $(\beta)$. Since $\sigma\left(T_{\epsilon_{k}}\right)$ has nonempty interior, $T_{\epsilon_{k}}$ has a nontrivial invariant subspace by [5, Theorem 2.1].

(ii) If $A$ and $B$ are hyponormal, then they are subscalar by [14], and so it is known that they have the property $(\beta)$. Hence it is obvious that $T_{\epsilon_{k}}$ has the property $(\beta)$ from Theorem 3.5.

(iii) Since $A$ and $B$ are compact or normal, then they are decomposable (see [10]) and this implies from Theorem 3.5 that $T_{\epsilon_{k}}$ is also decomposable.

From [2], if $T_{\epsilon_{k}}=\left(\begin{array}{cc}A & C \\ \epsilon_{k} D & B\end{array}\right)$ on $\mathcal{H} \oplus \mathcal{H}$ and $R(C)$ is closed, then we have the following matrix representation:

$$
T_{\epsilon_{k}}=\left(\begin{array}{ccc}
A_{1} & 0 & 0 \\
A_{2} & 0 & C_{1} \\
\epsilon_{k} D & B_{1} & B_{2}
\end{array}\right),
$$

which maps from $\mathcal{H} \oplus \mathcal{H}=\mathcal{H} \oplus N(C) \oplus N(C)^{\perp}$ into $\mathcal{H} \oplus \mathcal{H}=R(C)^{\perp} \oplus R(C) \oplus \mathcal{H}$ where $C_{1}=\left.C\right|_{N(C) \perp}, A_{1}=P_{R(C) \perp} A, A_{2}=P_{R(C)} A, B_{1}=\left.B\right|_{N(C)}$ and $B_{2}=\left.B\right|_{N(C) \perp}$. Here, $P_{N(C)}$ (resp. $P_{N(C) \perp}$ ) denotes the projection of $\mathcal{K}$ onto $N(C)$ (resp. $N(C)^{\perp}$ ). We now study the next theorem in the sense of the representation (9) and mention that a sequence $\left\{\epsilon_{k}\right\}$ need not converge to 0 .

Theorem 3.7 Let $T_{\epsilon_{k}}=\left(\begin{array}{cc}A & C \\ \epsilon_{k} D & B\end{array}\right)$ where $\left\{\epsilon_{k}\right\}$ is a bounded sequence and $R(C)$ is closed. Suppose that $A_{1}=P_{R(C)} A_{\left.\right|_{\mathcal{H}}}$ and $B_{1}=\left.B\right|_{N(C)}$. If $A_{1}$ has the property $(\beta)$ and $B_{1}$ is decomposable, then $T_{\epsilon_{k}}$ is decomposable. Moreover, if 0 is not an eigenvalue of $C^{*}$, then $T_{\epsilon_{k}}$ is decomposable if and only if $B_{1}$ is decomposable.

Proof Since $B_{1}$ is decomposable, both $B_{1}$ and $B_{1}{ }^{*}$ have the property $(\beta)$. Moreover, $A_{1}$ has the property $(\beta)$, thus $T_{\epsilon_{k}}$ and its adjoint operator have the property $(\beta)$ from [3, Theorem 3.3]. Therefore $T_{\epsilon_{k}}$ is decomposable. On the other hand, if $T_{\epsilon_{k}}$ is decomposable, then $T_{\epsilon_{k}}$ and its adjoint operator have the property $(\beta)$. Thus, by [3, Theorem 3.3], both $B_{1}$ and $B_{1}{ }^{*}$ have the property $(\beta)$. Hence $B_{1}$ is decomposable. The converse implication holds by a similar method. 
Corollary 3.8 Let $T_{\epsilon_{k}}=\left(\begin{array}{cc}A & C \\ \epsilon_{k} D & B\end{array}\right)$ where $\left\{\epsilon_{k}\right\}$ is a bounded sequence. If $C=\epsilon_{k} I$ and $A$ is selfadjoint, then $T_{\epsilon_{k}}$ is decomposable.

Proof Since $A$ is self-adjoint, so is $A_{1}=\left.P_{R(C)} \perp A\right|_{\mathcal{H}}$. Thus it has the property $(\beta)$. Since $\left\{\epsilon_{k}\right\}$ is a bounded sequence, $B_{1}=\left.B\right|_{N(C)}$ is decomposable, so it follows from Theorem 3.7 that $T_{\epsilon_{k}}$ is decomposable.

Example 3.9 Let $T_{\epsilon_{k}}=\left(\begin{array}{cc}A & U^{*} \\ \epsilon_{k} D & B\end{array}\right)$ on $\ell^{2}(\mathbb{N}) \oplus \ell^{2}(\mathbb{N})$ where $\left\{\epsilon_{k}\right\}$ is a bounded sequence and $U$ is the unilateral shift given by $U e_{n}=e_{n+1}$ on $\ell^{2}(\mathbb{N})$ for $n \in \mathbb{N}$. Then $B_{1}=\left.B\right|_{N(U)}$ is decomposable and this is equivalent to $T_{\epsilon_{k}}$ being decomposable by Theorem 3.7.

Now, we address Weyl type theorems for $T_{\epsilon_{k}}$. We start with the following lemma.

Lemma 3.10 Let $T_{\epsilon_{k}}=\left(\begin{array}{cc}A & C \\ \epsilon_{k} D & B\end{array}\right)$ where $A, B, C, D \in \mathcal{L}(\mathcal{H})$ and $\left\{\epsilon_{k}\right\}$ is a positive sequence such that $\lim _{k \rightarrow \infty} \epsilon_{k}=0$. Assume that $A$ and $B$ have the single-valued extension property. Then (a-)Browder's theorem holds for $T_{\epsilon_{k}}$.

Proof By Theorem 3.1, we know that $T_{\epsilon_{k}}$ has the single-valued extension property. Then it is obvious that $\sigma_{w}\left(T_{\epsilon_{k}}\right)=\sigma_{b}\left(T_{\epsilon_{k}}\right)$ and $\sigma_{e a}\left(T_{\epsilon_{k}}\right)=\sigma_{a b}\left(T_{\epsilon_{k}}\right)$ (see [1]). Hence this means that (a-)Browder's theorem holds for $T_{\epsilon_{k}}$.

Theorem 3.11 Let $T_{\epsilon_{k}}=\left(\begin{array}{cc}A & C \\ \epsilon_{k} D & B\end{array}\right)$ where $A, B, C, D \in \mathcal{L}(\mathcal{H})$ and $\left\{\epsilon_{k}\right\}$ is a positive sequence such that $\lim _{k \rightarrow \infty} \epsilon_{k}=0$. Suppose that Weyl's theorem holds for $A$ and $B$. Then the following statements hold.

(i) If $A$ and $B$ have the single-valued extension property, then Weyl's theorem holds for $T_{\epsilon_{k}}$.

(ii) If $A^{*}$ and $B^{*}$ have the single-valued extension property, then a-Weyl's theorem holds for $T_{\epsilon_{k}}$.

Proof (i) If $A$ and $B$ have the single-valued extension property, then it follows from Lemma 3.10 that $\sigma\left(T_{\epsilon_{k}}\right) \backslash \sigma_{w}\left(T_{\epsilon_{k}}\right)=p_{00}\left(T_{\epsilon_{k}}\right) \subseteq \pi_{00}\left(T_{\epsilon_{k}}\right)$. To show the reverse, we suppose that $0 \in \pi_{00}\left(T_{\epsilon_{k}}\right)$ without loss of generality. It follows from Theorem 3.4 that

$$
0 \in\left[\pi_{00}(A) \backslash \sigma(B)\right] \cup\left[\pi_{00}(B) \backslash \sigma(A)\right] \cup\left[\pi_{00}(A) \cap \pi_{00}(B)\right] .
$$

Since Weyl's theorem holds for both $A$ and $B$, we have $0 \notin \sigma_{w}(A) \cup \sigma_{w}(B)$. Set $T_{0}:=\left(\begin{array}{cc}A & C \\ 0 & B\end{array}\right)$. Then $T_{0}$ is Weyl by [11, Lemma 3]. This implies from [13, Theorem 1] that, if $T_{\epsilon_{k}} \rightarrow T_{0}$ in norm, then $\lim \sup _{k \rightarrow \infty} \sigma_{w}\left(T_{\epsilon_{k}}\right) \subset \sigma_{w}\left(T_{0}\right)$. Hence $0 \notin \lim \sup _{k \rightarrow \infty} \sigma_{w}\left(T_{\epsilon_{k}}\right)$. So there exists $\delta_{1}>0$ such that, for $\mu \in D\left(0, \frac{\delta_{1}}{2}\right)$, open disc with center 0 and radius $\frac{\delta_{1}}{2}$, such that $T_{\epsilon_{k}}-\mu I$ is Weyl. Since Weyl operators form an open set, there exists $\delta_{2}>0$ such that $\left\|T_{\epsilon_{k}}-\mu I-T_{0}\right\|<$ $\frac{\delta_{2}}{2}$. We choose $\delta:=\min \left\{\delta_{1}, \delta_{2}\right\}>0$. Then

$$
\left\|T_{\epsilon_{k}}-T_{0}\right\| \leq\left\|T_{\epsilon_{k}}-\mu I-T_{0}\right\|+\|\mu I\|<\frac{\delta}{2}+\frac{\delta}{2}=\delta .
$$

Therefore $T_{\epsilon_{k}}$ is Weyl but is not invertible. Consequently, Weyl's theorem holds for $T_{\epsilon_{k}}$. 
(ii) By Lemma 3.10, we have $\sigma_{a}\left(T_{\epsilon_{k}}\right) \backslash \sigma_{e a}\left(T_{\epsilon_{k}}\right)=p_{00}^{a}\left(T_{\epsilon_{k}}\right) \subseteq \pi_{00}^{a}\left(T_{\epsilon_{k}}\right)$. We now suppose that $0 \in \pi_{00}^{a}\left(T_{\epsilon_{k}}\right)$. From Theorem 3.4, we get

$$
0 \in\left[\pi_{00}^{a}(A) \backslash \sigma_{a}(B)\right] \cup\left[\pi_{00}^{a}(B) \backslash \sigma_{a}(A)\right] \cup\left[\pi_{00}^{a}(A) \cap \pi_{00}^{a}(B)\right] .
$$

It is known that $\sigma(S)=\sigma_{a}(S)$ and $\sigma_{w}(S)=\sigma_{e a}(S)$ provided $S^{*} \in \mathcal{L}(\mathcal{H})$ has the single-valued extension property by [1]. On the other hand, $A^{*}$ and $B^{*}$ have the single-valued extension property, and satisfy Weyl's theorem. This implies that $0 \notin \sigma_{w}(A) \cup \sigma_{w}(B)$. Then $T_{0}$ is Weyl. Hence we get $0 \notin \lim \sup _{k \rightarrow \infty} \sigma_{w}\left(T_{\epsilon_{k}}\right)$, so that $0 \in \sigma_{a}\left(T_{\epsilon_{k}}\right) \backslash \sigma_{e a}\left(T_{\epsilon_{k}}\right)$. Therefore $a$-Weyl's theorem holds for $T_{\epsilon_{k}}$.

We say that $T \in \mathcal{L}(\mathcal{H})$ is normal if $T^{*} T=T T^{*}$, hyponormal if $T^{*} T \geq T T^{*}$, algebraically hyponormal if there exists a nonconstant polynomial $p$ such that $p(T)$ is hyponormal, respectively. It is known that normal operators imply hyponormal operators, and hyponormal operators imply algebraically hyponormal operators. From these notions, we have the following corollary.

Corollary 3.12 Let $T_{\epsilon_{k}}=\left(\begin{array}{cc}A & C \\ \epsilon_{k} D & B\end{array}\right)$ where $A, B, C, D \in \mathcal{L}(\mathcal{H})$ and $\left\{\epsilon_{k}\right\}$ is a positive sequence such that $\lim _{k \rightarrow \infty} \epsilon_{k}=0$.

(i) If $A$ and $B$ are normal, then a-Weyl's theorem holds for $T_{\epsilon_{k}}$,

(ii) If $A$ and $B$ are algebraically hyponormal, then Weyl's theorem holds for $T_{\epsilon_{k}}$.

Proof (i) It is obvious that normal operators are decomposable by [10]. So if $A$ and $B$ are normal, then their adjoint operators have the single-valued extension property. Moreover, $A$ and $B$ are hyponormal, hence it follows from [4] that they satisfy Weyl's theorem. Consequently, this means that $a$-Weyl's theorem holds for $T_{\epsilon_{k}}$ from Theorem 3.11.

(ii) If $A$ and $B$ are algebraically hyponormal, then so are their translation, and then it follows from [6, Lemma 1] that $A-\lambda I$ and $B-\lambda I$ have finite ascent for all complex number $\lambda$, so that they have the single-valued extension property. On the other hand, Weyl's theorem holds for both $A$ and $B$ from [6, Corollary 4]. Thus this implies from Theorem 3.11 that $T_{\epsilon_{k}}$ satisfies Weyl's theorem.

Finally, we study $2 \times 2$ operator matrices

$$
T_{\gamma}=\left(\begin{array}{ll}
A & C \\
\gamma I & B
\end{array}\right)
$$

where $\gamma$ is any scalar in $\mathbb{C}$. Let $\left\{T_{\gamma}\right\}^{\prime}$ be the collection of operators commuting with $T_{\gamma}$ as follows:

$$
\left\{T_{\gamma}\right\}^{\prime}=\left\{\left(\begin{array}{cc}
L_{\sigma} & M_{\sigma} \\
N_{\sigma} & P_{\sigma}
\end{array}\right): \sigma \in \Sigma\right\},
$$

and let

$$
\left\{T_{\gamma}\right\}_{0}^{\prime}=\left\{\left(\begin{array}{cc}
L_{\sigma} & M_{\sigma} \\
N_{\sigma} & P_{\sigma}
\end{array}\right): \sigma \in \Sigma \text { and } \sup _{\sigma \in \Sigma}\left\|L_{\sigma}-P_{\sigma}\right\|<\infty\right\} .
$$


We recall that a transitive subalgebra of $\mathcal{L}(\mathcal{H})$ has the property that it has no nontrivial invariant subspace.

Theorem 3.13 Let $T_{\epsilon_{k}}=\left(\begin{array}{cc}A & C \\ \epsilon_{k} I & B\end{array}\right)$ where $A, B, C \in \mathcal{L}(\mathcal{H})$ and $\left\{\epsilon_{k}\right\}$ is a positive sequence such that $\lim _{k \rightarrow \infty} \epsilon_{k}=0$ and there is $X \in \mathcal{L}(\mathcal{H})$ such that $A X=X B$. If there exists a nontrivial hyperinvariant subspace $\mathcal{N}$ for $B$ such that $\mathcal{N} \not \subset \operatorname{ker} X$, then $S \in\left\{T_{\epsilon_{k}}\right\}_{0}^{\prime}$ has a nontrivial invariant subspace.

Proof Assume that there exists a nontrivial hyperinvariant subspace $\mathcal{N}$ for $B$ such that $\mathcal{N} \not \subset \operatorname{ker} X$. Let $S \in\left\{T_{\epsilon_{n}}\right\}_{0}^{\prime}$. Then we put $S=\left(\begin{array}{cc}L_{\sigma} & M_{\sigma} \\ N_{\sigma} & P_{\sigma}\end{array}\right)$ where $\sigma \in \Sigma$ and $\sup _{\sigma \in \Sigma}\left\|L_{\sigma}-P_{\sigma}\right\|<$ $\infty$. Since $S \in\left\{T_{\epsilon_{n}}\right\}_{0}^{\prime}$, we get

$$
\left(\begin{array}{ll}
L_{\sigma} A+\epsilon_{n} M_{\sigma} & L_{\sigma} C+M_{\sigma} B \\
N_{\sigma} A+\epsilon_{n} P_{\sigma} & N_{\sigma} C+P_{\sigma} B
\end{array}\right)=\left(\begin{array}{ll}
A L_{\sigma}+C N_{\sigma} & A M_{\sigma}+C P_{\sigma} \\
\epsilon_{n} L_{\sigma}+B N_{\sigma} & \epsilon_{n} M_{\sigma}+B P_{\sigma}
\end{array}\right) .
$$

Then we have $B N_{\sigma}-N_{\sigma} A=\epsilon_{n}\left(P_{\sigma}-L_{\sigma}\right)$ and so

$$
\left\|B N_{\sigma}-N_{\sigma} A\right\|=\epsilon_{n}\left\|P_{\sigma}-L_{\sigma}\right\| \leq \epsilon_{n} \sup _{\sigma \in \Sigma}\left\|P_{\sigma}-L_{\sigma}\right\|
$$

Since $\lim _{n \rightarrow \infty} \epsilon_{n}=0, B N_{\sigma}=N_{\sigma} A$ for $\sigma \in \Sigma$. Hence $B N_{\sigma} X=N_{\sigma} A X=N_{\sigma} X B$ for $\sigma \in \Sigma$. So $N_{\sigma} X \mathcal{N} \subset \mathcal{N}$ for $\sigma \in \Sigma$. On the other hand, assume, to obtain a contradiction, that $\left\{T_{\epsilon_{n}}\right\}_{0}^{\prime}$ is transitive. Then, for arbitrary $z \in \mathcal{H}$, it follows from [7, Proposition 2.2] and the hypothesis that there exist $\sigma_{0} \in \Sigma$ and $y \in \mathcal{N}$ with $X y \neq 0$ such that, for every $\epsilon>0$,

$$
\left\|N_{\sigma_{0}} X y-z\right\|<\epsilon,
$$

which means that $\overline{\left\{\mathcal{N}_{\sigma} X y: \sigma \in \Sigma\right\}}=\mathcal{H}$ for some $\sigma_{0} \in \Sigma$. But this is a contradiction from $N_{\sigma} X y \in \mathcal{N}$ for all $\sigma \in \Sigma$. Hence $\left\{T_{\epsilon_{n}}\right\}_{0}^{\prime}$ is not transitive. Thus $S \in\left\{T_{\epsilon_{n}}\right\}_{0}^{\prime}$ has nontrivial invariant subspace.

We easily see that there exists a nontrivial hyperinvariant subspace $\mathcal{N}$ for $B$ such that $\mathcal{N} \nsubseteq \operatorname{ker} X$ as the following example.

Example 3.14 Let $N \in \mathcal{L}(\mathcal{H})$ be a normal operator with $N \neq \lambda I$ for $\lambda \in \mathbb{C}$. Consider an operator matrix $\left(\begin{array}{cc}N & C \\ \epsilon_{k} I & N\end{array}\right)$ and $\left\{\epsilon_{k}\right\}$ is a positive sequence such that $\lim _{k \rightarrow \infty} \epsilon_{k}=0$. Then there exists a nontrivial hyperinvariant subspace $\mathcal{N}$ for $N$ but $\mathcal{N} \nsubseteq \operatorname{ker} I$. Hence $S \in\left\{\left(\begin{array}{cc}N & C \\ \epsilon_{k} I & N\end{array}\right)\right\}_{0}^{\prime}$ has a nontrivial invariant subspace by Theorem 3.13.

Corollary 3.15 Let $T_{\delta_{k}}=\left(\begin{array}{cc}A & \delta_{k} I \\ Z & B\end{array}\right)$ where $A, B, Z \in \mathcal{L}(\mathcal{H})$ and $\delta_{k}$ is a positive sequence such that $\lim _{n \rightarrow \infty} \delta_{k}=0$ and there is $X \in \mathcal{L}(\mathcal{H})$ such that $B X=X A$. If there exists a nontrivial hyperinvariant subspace $\mathcal{M}$ for $A$ such that $\mathcal{M} \not \subset \operatorname{ker} X$, then $S \in\left\{T_{\delta_{k}}\right\}_{0}^{\prime}$ has nontrivial invariant subspace.

Proof Set $R_{\delta_{k}}=\left(\begin{array}{cc}B & Z \\ \delta_{k} I & A\end{array}\right)$. Since $\left(\begin{array}{cc}0 & I \\ I & 0\end{array}\right) R_{\delta_{k}}\left(\begin{array}{cc}0 & I \\ I & 0\end{array}\right)=T_{\delta_{k}}, R_{\delta_{k}}$ and $T_{\delta_{k}}$ are unitarily equivalent. Since $W \in\left\{R_{\delta_{k}}\right\}_{0}^{\prime}$ has a nontrivial invariant subspace by Theorem 3.13,

$$
\left(\begin{array}{ll}
0 & I \\
I & 0
\end{array}\right) W\left(\begin{array}{cc}
0 & I \\
I & 0
\end{array}\right) T_{\delta_{k}}=\left(\begin{array}{cc}
0 & I \\
I & 0
\end{array}\right) W R_{\delta_{k}}\left(\begin{array}{ll}
0 & I \\
I & 0
\end{array}\right)
$$




$$
\begin{aligned}
& =\left(\begin{array}{ll}
0 & I \\
I & 0
\end{array}\right) R_{\delta_{k}} W\left(\begin{array}{ll}
0 & I \\
I & 0
\end{array}\right) \\
& =T_{\delta_{k}}\left(\begin{array}{ll}
0 & I \\
I & 0
\end{array}\right) W\left(\begin{array}{ll}
0 & I \\
I & 0
\end{array}\right) .
\end{aligned}
$$

Thus $\left(\begin{array}{cc}0 & I \\ I & 0\end{array}\right) W\left(\begin{array}{cc}0 & I \\ I & 0\end{array}\right) \in\left\{T_{\delta_{k}}\right\}^{\prime}$. Since $W \in\left\{R_{\delta_{k}}\right\}_{0}^{\prime}$,

$$
\left(\begin{array}{ll}
0 & I \\
I & 0
\end{array}\right) W\left(\begin{array}{cc}
0 & I \\
I & 0
\end{array}\right) \in\left\{T_{\delta_{k}}\right\}_{0}^{\prime}
$$

Since $W \in\left\{R_{\delta_{k}}\right\}_{0}^{\prime}$ has a nontrivial invariant subspace, we conclude that

$$
S=\left(\begin{array}{ll}
0 & I \\
I & 0
\end{array}\right) W\left(\begin{array}{ll}
0 & I \\
I & 0
\end{array}\right) \in\left\{T_{\delta_{k}}\right\}_{0}^{\prime}
$$

has a nontrivial invariant subspace.

\section{Acknowledgements}

The authors would like to thank the referee for his/her valuable comments, which helped to improve the paper.

\section{Funding}

II Ju An was supported by the National Research Foundation of Korea (NRF) grant funded by the Korea government (MSIT) (2020R1A2C1A01006036). Eungil Ko was supported by the National Research Foundation of Korea (NRF) grant funded by the Korea government (MSIT) (2019R1F1A1058633) and the Ministry of Education (2019R1A6A1A11051177). Ji Eun Lee was supported by Basic Science Research Program through the National Research Foundation of Korea (NRF) funded by the Ministry of Education, Science and Technology (2019R1A2C1002653).

\section{Availability of data and materials}

No data were used to support this study.

\section{Competing interests}

The authors declare that they have no competing interests.

\section{Authors' contributions}

All authors contributed equally to the writing of this paper. All authors read and approved the final manuscript.

\section{Author details}

${ }^{1}$ Department of Applied Mathematics, Kyung Hee University, Yongin, Gyeonggi-do 17104, Korea. ${ }^{2}$ Department of Mathematics, Ewha Womans University, Seoul, 120-750, Korea. ${ }^{3}$ Department of Mathematics and Statistics, Sejong University, Seoul, 143-747, Korea.

\section{Publisher's Note}

Springer Nature remains neutral with regard to jurisdictional claims in published maps and institutional affiliations.

Received: 10 June 2021 Accepted: 18 September 2021 Published online: 02 October 2021

\section{References}

1. Aiena, P.: Fredholm and Local Spectral Theory with Applications to Multipliers. Kluwer Academic, Dordrecht (2004)

2. Alatancang, Hou, G., Hai, G.: Perturbation of spectra for a class of $2 \times 2$ operator matrices. Acta Math. Appl. Sin. 28, $711-720(2012)$

3. An, I.J., Ko, E., Lee, J.E.: Properties of operator matrices. J. Korean Math. Soc. 57(4), 893-913 (2020)

4. Coburn, L.A.: Weylâ s theorem for nonnormal operators. Mich. Math. J. 13, 285-288 (1966)

5. Eschmeier, J.: Invariant subspaces for operators with Bishop's property $(\beta)$ and thick spectrum. J. Funct. Anal. 94 , 196-222 (1990)

6. Han, Y.M., Lee, W.Y.: Weyl's theorem holds for algebraically hyponormal operators. Proc. Am. Math. Soc. 128(8), $2291-2296(2000)$

7. Jung, I.B., Ko, E., Pearcy, C.: Hyperinvariant subspaces for some $2 \times 2$ operator matrices. Kyungpook Math. J. 58(3), 489-494 (2018)

8. Kim, H.J.: Hyperinvariant subspaces for operators having a normal part. Oper. Matrices 5, 487-494 (2011)

9. Ko, E.: Local spectral property of $2 \times 2$ operator matrices. Filomat 33(7), 1845-1854 (2019) 
10. Laursen, K., Neumann, M.: An Introduction to Local Spectral Theory. Clarendon Press, Oxford (2000)

11. Lee, W.Y.: Weyl spectra of operator matrices. Proc. Am. Math. Soc. 129, 131-138 (2000)

12. Lee, W.Y.: Lecture Notes on Operator Theory. Seoul National University, Seoul (2010)

13. Oberai, K.K.: On the Weyl spectrum. III. J. Math. 18, 208-212 (1974)

14. Putinar, M.: Hyponormal operators are subscalar. J. Oper. Theory 12, 385-395 (1984)

Submit your manuscript to a SpringerOpen ${ }^{\circ}$ journal and benefit from:

- Convenient online submission

$\checkmark$ Rigorous peer review

- Open access: articles freely available online

- High visibility within the field

Retaining the copyright to your article

Submit your next manuscript at $\gg$ springeropen.com 